\title{
苯并噻二唑类电子受体材料分子设计与给-受体的理论匹配
}

\author{
邵线 杨金金博 尹世伟 王文亮* \\ (陕西省大分子科学重点实验室 陕西师范大学化学化工学院 西安 710119)
}

\begin{abstract}
摘要 设计并理论预测了一系列 $\mathrm{A}^{\prime}-\pi-\mathrm{A}-\pi-\mathrm{A}^{\prime}$ 型苯并噻二唑衍生物电子受体分子的几何构型、前线轨道特征、吸光性质 及电子重组能等信息，同时考察了丙酮、氯苯溶剂对其性质的影响. 在此基础上将性能优异的受体分子与特定给体分 子组合构建给-受体(D-A)界面, 通过计算给体 HOMO 与受体 LUMO 之间的电子耦合 $V_{\mathrm{if}}$, 评估了 D-A 界面载流子的复 合程度. 结果表明, 合理选择取代基对核心受体苯并噻二唑进行修饰是调节 LUMO 能级和能隙的有效方法. 将平面性 好的受体材料与非平面型给体材料搭配作为有机太阳能电池光活性层材料, 有可能达到降低界面复合、减小光电压损 失和提高开路电压的目的. 综合考虑 $\Delta E_{\mathrm{L}}, V_{\mathrm{if}}$, 光吸收效率及溶剂化效应等因素, D1-1a $\gamma$ 及 D1-2a $\gamma$ 组合有望成为电子迁 移率高、在可见光和近红外区吸收宽、界面激子可以有效分离且不易复合的理想给-受体分子组合.

关键词 苯并噻二唑; 电子受体分子; 给-受体界面; 电子耦合; 能量转换效率
\end{abstract}

\section{Molecular Design of Benzothiadiazole Derivatives Electron Acceptors and Matching of Donor-Acceptor Materials}

\author{
Shao, Rong Yang, Xinbo Yin, Shiwei Wang, Wenliang* \\ (Key Laboratory for Macromolecular Science of Shaanxi Province, School of Chemistry \& Chemical Engineering, Shaanxi \\ Normal University, Xi'an 710119, China)
}

\begin{abstract}
To better understand the relationships between the microstructure and the optoelectronic characteristics of the electron acceptor and to meet the needs of donor-acceptor materials with excellent optical properties for solar cell, a series of acceptor molecules with $\mathrm{A}^{\prime}-\pi-\mathrm{A}-\pi-\mathrm{A}^{\prime}$ type are designed. In these molecules, the core framework of benzothiadiazole is used as an acceptor (A), three kinds of conjugated heterocyclics (A') with different abilities of electron-withdrawing and steric effects are applied as the terminals, and various conjugated structures, such as the double bond, thiophene, benzothiophene and vinyl thiophene, are utilized as $\pi$-bridge, respectively. Their geometric configurations, the characteristics of frontier molecular orbital, optical properties, as well as the electronic reorganization energy are predicted by DFT-B3LYP and TD-DFT-CAM-B3LYP. Solvent effects from acetone and chlorobenzene on molecular properties are studied. Furthermore, the Donor-Acceptor (D-A) interfaces are respectively constructed by combining the excellent acceptors with the selected two donors. The DFT-D3 method is used to scan the binding energy of D-A complex, in order to determine the stacked displacement of the interface. The degree of interface recombination is evaluated by calculating electronic coupling $\left(V_{\text {if }}\right)$ between HOMO of donors and LUMO of acceptors. The results show that modifying benzothiadiazole with a reasonable substituent is an effective way to adjust LUMO energy levels and lead to the noticeable variation of the energy gap. Combining planar electron acceptor materials ( $\mathrm{A}^{\prime}-\pi-\mathrm{A}-\pi-\mathrm{A}^{\prime}$ type) with non-planar electron donor materials (D), to form the optical active layer is a practical approach for preventing interface recombination and achieving high open-circuit voltage $\left(V_{\text {oc }}\right)$. Considering $\Delta E_{\mathrm{L}}, V_{\text {if }}$, light absorption efficiency, and solvation effect, D1-1a $\gamma$ and D1-2a $\gamma$ combinations are the most promising candidates of optical active layer materials in organic solar cell.

Keywords benzothiadiazole derivatives; electronic acceptors; Donor-Acceptor interface; electronic coupling; the power conversion efficiency
\end{abstract}

\section{1 引言}

有机太阳能电池(OPV)以其环保、低成本等诸多优 势受到研究者们的青睐 ${ }^{[1 \sim 3]}$. 有机太阳能电池的工作过 程可以分为四步: (1) 有机光活性层吸收光子, 产生激 子(电子-空穴对). 光吸收效率 $\eta_{\mathrm{A}}$ 与光活性层在可见光
区的吸收密切相关; (2) 激子在光活性层中向给-受体 (D-A) 界面处扩散. 激子扩散效率 $\eta_{\mathrm{ED}}$ 与有机光活性层材 料的载流子迁移率等因素有关; (3) 扩散到 D-A 界面的 激子克服束缚能, 分离成自由空穴和自由电子. 从电子 结构上看, 激子分离效率 $\eta_{\mathrm{CT}}$ 与给-受体的 LUMO 能级

\footnotetext{
*E-mail: wlwang@snnu.edu.cn

Received May 30, 2016; published July 18, 2016.
}

Supporting information for this article is available free of charge via the Internet at http://sioc-journal.cn.

Project supported by the National Natural Science Foundation of China (Nos. 21173139, 21473108) and Shaanxi Innovative Team of Key Science and Technology (No. 2013KCT-17).

项目受国家自然科学基金(Nos. 21173139, 21473108)和陕西省重点科技创新团队基金(No. 2013KCT-17)资助. 
之差 $\Delta E_{\mathrm{L}}$ 有关; (4) 自由空穴和自由电子在内建电场的 作用下分别被阳极和阴极所收集. 载流子收集效率 $\eta_{\mathrm{CC}}$ 主要受载流子迁移率和各种复合的影响, 其中 D-A 界面 的载流子复合会造成光电压损失, 不利于获得高的开路 电压 $V_{\mathrm{oc}}$. D-A 界面载流子的复合程度可以用给体的 $\mathrm{HOMO}\left(\mathrm{HOMO}^{\mathrm{D}}\right)$ 与受体的 $\mathrm{LUMO}\left(\mathrm{LUMO}^{\mathrm{A}}\right)$ 之间的电子 耦合 $V_{\text {if }}$ 来度量 ${ }^{[4]}$. 这四步所对应的效率共同决定了 OPV 器件能量转换效率 $\eta$ 的大小 ${ }^{[5 \sim 8]}$. 可见, 改善有机 光活性层材料分子性能, 减小 D-A 界面载流子复合对提 高 OPV 器件能量转换效率非常重要.

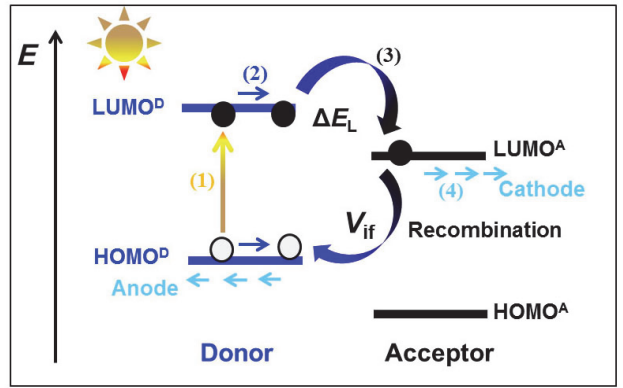

图式 1 有机太阳能电池工作原理示意图

Scheme 1 The working principle diagram of organic photovoltaic cells

近年来受体材料分子的研究主要集中在富勒烯及 其衍生物上, 然而富勒烯存在可见光区吸收范围窄、能 级可调性差等缺陷, 人们开始将视角转向非富勒烯电子 受体材料的研究 ${ }^{[9 \sim 11]}$. 苯并噻二唑衍生物由于制备方法 多样、易于得到高度有序薄膜获得良好的电子传输性能 而成为受体材料分子候选者受到了广泛的关注 ${ }^{[12 ~ 15] . ~}$

基于上述分析，本文以实验合成分子 $\mathbf{1 a \alpha}$ 为基 础 ${ }^{[15]}$, 设计了一系列 $\mathrm{A}^{\prime}-\pi-\mathrm{A}-\pi-\mathrm{A}^{\prime}$ 型苯并噻二唑衍生物 电子受体分子 (Scheme 2), 利用量子化学计算方法及 CPCM 模型 ${ }^{[16]}$ 评估了相关性质; 将性能优异的受体分子 与选定的给体分子 D1 及 D2 匹配，构建 D-A 界面模型， 通过计算 $\mathrm{HOMO}^{\mathrm{D}}$ 与 $\mathrm{LUMO}^{\mathrm{A}}$ 间的电子耦合 $V_{\text {if }}$, 评估了 D-A 界面载流子的复合趋势; 提出了光电性能匹配有可 能获得较高开路电压的 D-A 分子组合, 以期为开发高性 能的有机太阳能电池提供理论指导.

\section{2 方法与原理}

Schlenker 等 ${ }^{[17]}$ 从给-受体材料自身性质出发对有机 太阳能电池光电压的损失进行了研究, 认为开路电压与 载流子复合速率成反比关系:

$$
V_{\mathrm{oc}} \approx \frac{n k_{\mathrm{B}} T}{q} \ln \left(\frac{J_{\mathrm{sc}}}{q k\left[D^{+} A^{-}\right]}\right)
$$

式中理想因子 $n$ 主要与暗电流-电压曲线有关, 对于有 机半导体材料通常 $n \approx 2, k_{\mathrm{B}}$ 为 Boltzmann 常数, $T$ 为热力
学温度, $J_{\mathrm{SC}}$ 是短路电流密度, $\left[\mathrm{D}^{+} \mathrm{A}^{-}\right]$是激子在 $\mathrm{D}-\mathrm{A}$ 界面 的浓度, $q$ 为元电荷, $k$ 为载流子复合速率常数.

根据 Marcus-Hush 理论 ${ }^{[18]}$, 载流子复合速率常数 可表示为 ${ }^{[19,20]}$ :

$$
k=\frac{4 \pi^{2}}{h} V_{\text {if }}^{2} \frac{1}{\sqrt{4 \pi \lambda k_{\mathrm{B}} T}} \exp \left\{-\frac{\left(\lambda+\Delta G_{0}\right)^{2}}{4 \lambda k_{\mathrm{B}} T}\right\}
$$

式中 $k_{\mathrm{B}}$ 与 $h$ 分别为 Boltzmann 和 Planck 常数, $T$ 为热力 学温度, $V_{\text {if }}$ 为 $\mathrm{HOMO}^{\mathrm{D}}$ 与 $\mathrm{LUMO}^{\mathrm{A}}$ 之间的电子耦合 ${ }^{[21]}, \lambda$ 为重组能, $\Delta G_{0}$ 是载流子复合过程的自由能变化值. 结 合 (1)与(2)式可知, 可通过减小 $V_{\text {if }}$ 来降低 D-A 界面的 载流子复合速率，达到提高有机太阳能电池开路电压 $V_{\mathrm{oc}}$ 的目的.

分子重组能包括外重组能和内重组能, 通常外重组 能较小, 可以不予考虑. 内重组能是指电荷载流子转移 前后, 分子总的结构弛豫能变化, 它是影响分子载流子 迁移率大小的重要因素. 因本文主要关注受体分子的电 子迁移率，采用如下公式计算电子重组能 $\lambda_{\mathrm{e}}$ :

$$
\lambda_{\mathrm{e}}=\left[E^{0}(-)-E^{0}(0)\right]+\left[E^{-}(0)-E^{-}(-)\right]
$$

式中 $E^{-}(0)$ 是中性分子几何构型下阴离子能量, $E^{-}(-)$是 优化构型后的阴离子能量, $E^{0}(0)$ 是中性分子能量, $E^{0}(-)$ 是中性分子在阴离子几何构型下能量 ${ }^{[22]}$.

以给体 $\mathrm{D}$ 与受体 $\mathrm{A}$ 分子之间的面对面堆积方式为 D-A 界面的初始构型, 通过坐标扫描方法获得稳定界面 复合物(D-A)的最终构型，采用孤立轨道法 ${ }^{[23]}$ 计算该构 型下的电子耦合 $V_{\text {if }}$ (计算原理与细节参见文献[23]), 不 同之处仅是本文选取 $\mathrm{HOMO}^{\mathrm{D}}$ 与 $\mathrm{LUMO}^{\mathrm{A}}$ 进行计算 $V_{\mathrm{if}}$. 采用 DFT-B3LYP/6-311G(d,p)方法对单分子几何构型进 行优化; 基于 TD-DFT 原理，在 CAM-B3LYP 和 B3LYP/6-311G $(d, p)$ 水平上预测受体分子的吸收光谱, 采用 DFT-D3 方法计算 D-A 复合物的结合能 $\Delta E_{\mathrm{b}}{ }^{[24 ~ 26]}$, 并参照文献[25～27]推荐的结合能扫描方法确定 D-A 界 面几何信息. 所有计算均采用 Gaussian 09 程序包完成.

\section{3 结果与讨论}

\section{1 受体分子设计思想}

基于实验上合成的苯并噻二唑衍生物分子 $\mathbf{1 a \alpha}$, 设 计了一系列 $\mathrm{A}^{\prime}-\pi-\mathrm{A}-\pi-\mathrm{A}^{\prime}$ 型电子受体分子, 结构示于 Scheme 2. 设计思想如下: (1) 以三种吸电子能力和位 阻不同的共轭杂环化合物 $1,2,3$ 为终端受体 $\mathrm{A}^{\prime}$, 分别以 双键 $\mathrm{a}$ 、噻吩 $\mathrm{b}$ 、并噻吩 $\mathrm{c}$ 及乙烯噻吩 $\mathrm{d}$ 为 $\pi$ 共轭桥, 考 察不同的终端受体和 $\pi$-桥对分子性质的影响; (2) 用不 同取代基 $\mathrm{R}$ 修饰核心受体 $\mathrm{A}$ ，进一步调节分子的光电性 质; (3) 保持分子对称结构，以降低 D-A 复合物的基态 偶极矩 ${ }^{[27]}$. 


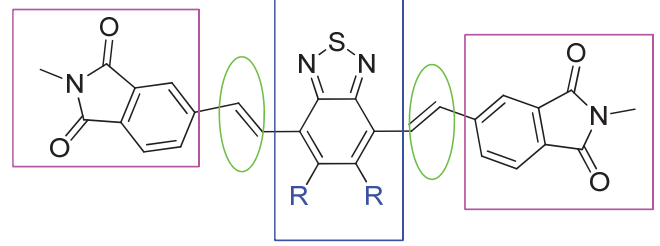

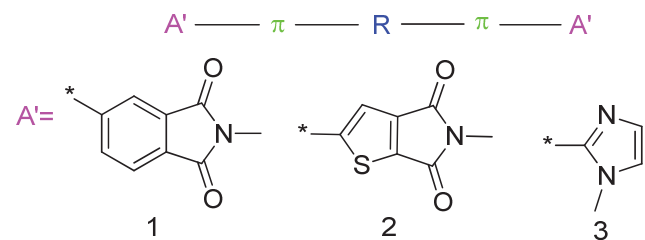

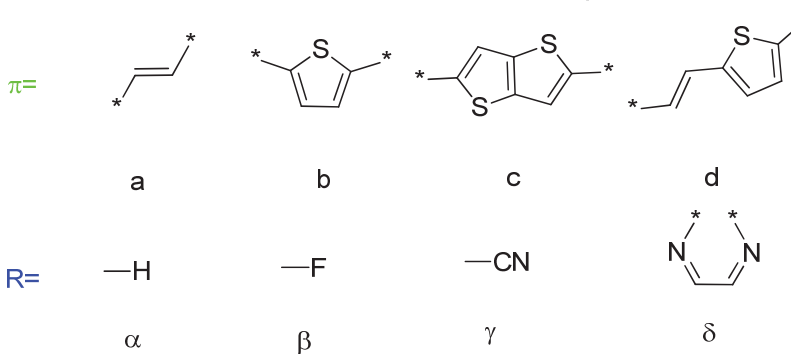

图式 2 苯并噻二唑衍生物电子受体的分子结构

Scheme 2 Chemical structures of electron acceptors containing benzothiadiazole

\section{2 受体分子几何构型及前线轨道能级}

平面分子构型倾向于 $\pi-\pi$ 紧密堆积, 有利于获得良 好的电子传输能力. 因此本文选择如图 1 所示的扭转角 $\theta_{1}(\mathrm{C} 1-\mathrm{C} 2-\mathrm{C} 3-\mathrm{C} 4)$ 和 $\theta_{2}$ (C5-C6-C7-C8)对分子的平面性进 行评估. 表 1 数据表明, $\pi$-桥不同时平面性次序为 $\pi-\mathrm{a}>$ $\pi-\mathrm{d}>\pi-\mathrm{b}>\pi-\mathrm{c} . \pi-\mathrm{b}$ 和 $\pi-\mathrm{c}$ 系列分子的 $\theta_{1}$ 分别为 $10.31^{\circ}$ 和 $6.72^{\circ}$, 而 $\pi-\mathrm{a}$ 和 $\pi-\mathrm{d}$ 系列分子的 $\theta_{1}$ 最大分别仅有 $0.02^{\circ}$ 和 $2.29^{\circ}$. 显然, $\pi-b$ 和 $\pi-c$ 系列分子平面性较差, 在下面 研究中予以排除, 相关扭转角 $\theta_{1}$ 和 $\theta_{2}$ 数据列在表 $\mathrm{S} 1$ 中.
$\mathrm{A}^{\prime}$ 不同时平面性次序为 $\mathrm{A}^{\prime}-3>\mathrm{A}^{\prime}-2>\mathrm{A}^{\prime}-1$, 其中与 $\mathrm{A}^{\prime}-2$ 相比, $A^{\prime}-1$ 由于较大的位阻效应使得分子平面性降低; $\mathrm{A}^{\prime}-3$ 系列分子中, $\mathbf{3 c \alpha}$ 分子出现扭转, 其余分子近乎平 面, 这可能是并噻吩刚性较强所致. $\pi$-a 和 $\pi$-d 系列分子 的核心受体 $\mathrm{A}$ 用 $\beta, \gamma, \delta$ 三个取代基取代结果显示，取代 基 $\mathrm{R}$ 空间位阻将 $\pi$-桥和 $\mathrm{A}^{\prime}$ 向上推，分子水平弯曲弧度变 大, $\theta_{1}$ 显著减小, $\theta_{2}$ 几乎不变, 分子平面性有所提高.

OPV 器件常采用具有高功函的金属 $(\mathrm{Al}: 4.26 \mathrm{eV}$, $\mathrm{Ag}$ : $4.28 \mathrm{eV}$ )等作为阳极材料, 并且为了能与多数给体 能级匹配，受体分子应具备较低的 LUMO 能级. 对于本 文选定的低聚噻吩衍生物给体分子 Dn, LUMO 能级在 $-3.3 \sim-3.6 \mathrm{eV}$ 之间，为了使给体与受体之间的 LUMO 能级差保持大于 $0.3 \mathrm{eV}$, 受体分子的 LUMO 能级 最好在 $3.6 \sim 4.1 \mathrm{eV}^{[28]}$ 之间. 计算结果表明, B3LYP/6-311G $(d, p)$ 方法得到 $\mathbf{1 a \alpha}$ 分子的 HOMO 能级值 $-5.97 \mathrm{eV}$, 与实验上采用 PESA (photoelectron spectroscopy in air)方法获得数值 $-6.05 \mathrm{eV}$ 十分接近 ${ }^{[15]}$. $\mathrm{A}^{\prime}$ 不同 时, LUMO 能级次序为 $\mathrm{A}^{\prime}-3>\mathrm{A}^{\prime}-1>\mathrm{A}^{\prime}-2$, 用 $\beta, \gamma, \delta$ 进一 步调节结果显示 $\mathrm{A}^{\prime}-3$ 系列分子 LUMO 能级仍然过高, 在后续研究中将不予考虑, 图 1 仅给出满足能级要求的 部分苯并噻二唑衍生物前线轨道能级, 其余数据示于图 S1 中. 同时发现 $\beta$ 使分子 LUMO 能级降低程度小于 HOMO 能级, 能隙增大; $\gamma$ 使分子 LUMO 能级降低程度 大于 HOMO 能级, 能隙减小; 可成环的取代基 $\delta$ 增加了 分子的共轭程度, 使分子 LUMO 能级降低, HOMO 能级 升高，能隙减小; 因此，合理选择取代基对核心受体苯 并噻二唑进行修饰是调节 LUMO 能级和能隙最有效的 方法. 综上分析，受体分子 $\mathbf{1 a} \gamma, \mathbf{1 a} \delta, 2 \mathrm{a} \gamma, \mathbf{2} \mathbf{a} \delta, \mathbf{1 d} \gamma, \mathbf{1 d} \delta$, $2 \mathrm{~d} \gamma$ 和 $2 \mathrm{~d} \boldsymbol{\delta}$ 初步满足受体分子平面性好、 LUMO 能级合 理的要求.

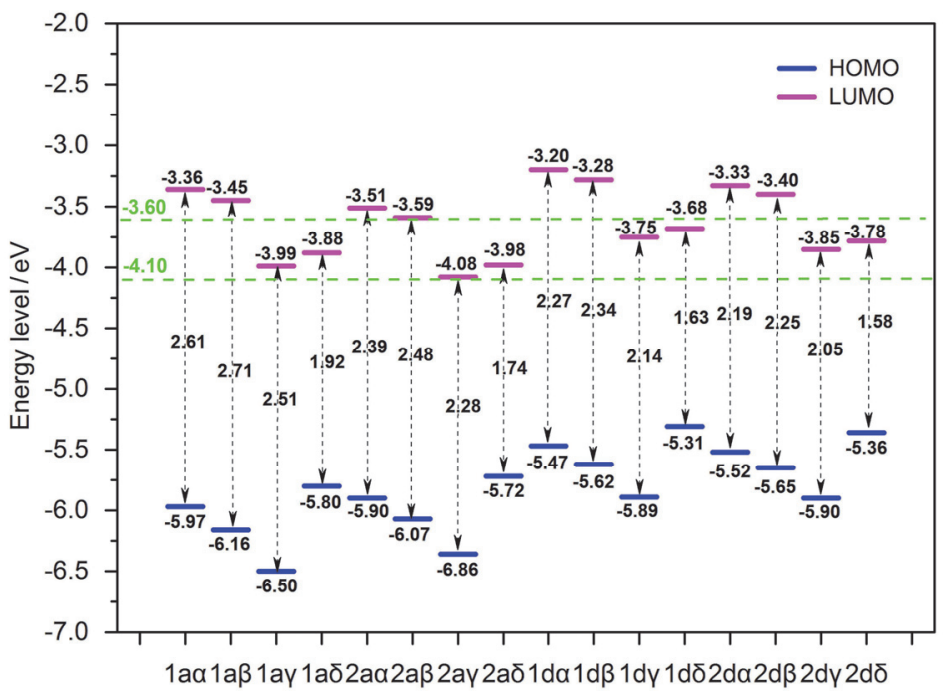

图 1 B 3 LYP/6-311G $(\mathrm{d}, \mathrm{p})$ 水平上获得部分苯并噻二唑衍生物前线轨道能级

Figure 1 HOMO and LUMO energy levels of part of benzothiadiazole derivatives calculated at B3LYP/6-311G(d,p) level 


\section{3 受体分子吸光性质与电子重组能}

为了提高太阳能电池的光吸收效率 $\eta_{\mathrm{A}}$, 给体和受 体材料在可见光和近红外区的吸收应尽可能互补, 以拓 宽光活性层的光谱吸收范围. 因此, 采用光谱吸收范围 和光捕获效率(LHE) 对受体材料的吸光性能进行评价, 其中 LHE 与振子强度 $f$ 成正比例关系可表示为 ${ }^{[29]}$ :

$$
\mathrm{LHE}=1-10^{-f}
$$

分子的最大吸收波长 $\lambda_{\max }$ 、振子强度 $f$ 、激发能 $E$ 列于表 2. 对唯一有光谱实验数据的 $\mathbf{1 a \alpha}$, 其 CAM-B3LYP 计算与实验 $\lambda_{\text {max }}$ 均为 $448 \mathrm{~nm}$. 从表 2 可知, B3LYP 比 CAM-B3LYP 方法普遍低估了激发能. 因此, 本文关于光谱与激发能讨论采用 CAM-B3LYP 方法计 算数据. 化合物 $\mathbf{1 a \delta}$ 和 $\mathbf{2} \mathbf{a} \boldsymbol{\delta}$ 的振子强度较小, 光捕获效 率较差; $1 \mathbf{d} \boldsymbol{\delta}$ 和 $\mathbf{2} \mathbf{d} \boldsymbol{\delta}$ 的最大吸收波长较长, 几乎超出可见 光和近红外区. 所以上述 4 个化合物在后续研究中不予 考虑.

此外, 电子重组能 $\lambda_{\mathrm{e}}$ 是影响受体分子电子迁移率的 主要参数之一, $\lambda_{\mathrm{e}}$ 越小, 电子迁移率越大. 表 2 同时列出 了采用绝热势能面法 B3LYP/6-311G(d,p)水平上计算的 $\lambda_{\mathrm{e}}$. 显然, 取代基 $\beta$ 对分子 $\lambda_{\mathrm{e}}$ 影响不大, 取代基 $\gamma$ 和 $\delta$ 使 分子的 $\lambda_{\mathrm{e}}$ 减小, 尤其是 $\gamma$, 这可能是由于 $\gamma$ 平衡了分子间 张力, 增强了分子的刚性. 分子 $1 \mathrm{a} \gamma, 2 \mathrm{a} \gamma, \mathbf{1 d} \gamma, 2 \mathrm{~d} \gamma$ 和 $2 \mathbf{d} \boldsymbol{\delta}$ 的重组能均小于 $0.200 \mathrm{eV}$, 有望获得较高的电子迁
移率.

综合考虑光谱吸收范围、振子强度 $f$ 及 $\lambda_{\mathrm{e}}$ 三个参数, 发现分子 $1 \mathrm{a} \gamma, 2 \mathrm{a} \gamma, \mathbf{1 d} \gamma$ 和 $2 \mathrm{~d} \gamma$ 具有较好的光电性能, 值 得实验上合成研究, 并模拟了这 4 个分子在气相及丙酮 和氯苯溶剂中的吸收光谱, 结果列于表 3 , 其中图 3 仅 示丙酮溶剂中的变化情况.

\section{4 溶剂化效应与受体分子相关性质}

采用 CPCM 模型研究了丙酮和氯苯溶剂对 $\mathbf{1 a} \gamma$, $\mathbf{2 a} \gamma, \mathbf{1 d} \gamma$ 和 $2 \mathrm{~d} \gamma$ 分子几何构型、吸光性质和电子重组能 的影响. 结果显示，以双键为 $\pi$-桥，氰基为取代基的 $1 \mathrm{a} \gamma$ 在溶剂中几何构型出现大幅度扭转, 分子平面性变差; 仅终端受体不同的 $2 \mathrm{a} \gamma$ 分子在不同溶剂中几何构型基本 不变. 这可能是由于 $1 \mathrm{a}$ 与 与溶剂分子形成氢键位点分布 在分子一侧, 加之终端受体 $\mathrm{A}^{\prime}-1$ 位阻较大, 致使双键 $\pi$ 桥发生扭转, $\mathrm{A}^{\prime}-2$ 上的 $\mathrm{S}$ 原子也可以形成 $\mathrm{S} \cdots \mathrm{H}$ 键, 氢键 位点分布在分子两侧, 使得 $2 \mathrm{a}$ \% 几何构型基本不变.

从光谱模拟图 2 看出，溶剂化效应使吸收强度明显 增强, 光谱吸收范围也显著展宽. 计算结果显示, $1 \mathrm{~d} \gamma$ 和 $\mathbf{2 d} \gamma$ 在两种溶剂中均发生红移, 而 $1 \mathrm{a} \gamma$ 在 2 种溶剂中发 生不同程度的蓝移. $2 \mathrm{a} \gamma$ 在丙酮中稍有蓝移，但在氯苯 中却稍有红移. 这些移动均在可见光和近红外区, 并不 影响分子总体吸光性质. 表 3 重组能 $\lambda_{\mathrm{e}}$ 数据表明, 在溶 剂中受体 $1 \mathrm{a} \gamma$ 分子 $\lambda_{\mathrm{e}}$ 稍有增大, 而 $2 \mathrm{a} \gamma, \mathbf{1 d} \gamma$ 与 $2 \mathrm{~d} \gamma$ 的 $\lambda_{\mathrm{e}}$

表 1 部分苯并噻二唑衍生物受体分子几何结构参数

Table 1 Geometry parameters for the description of benzothiadiazole derivatives

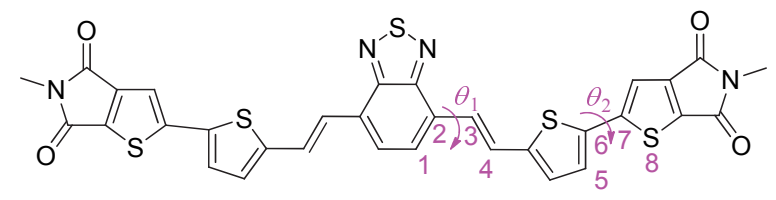

\begin{tabular}{|c|c|c|c|c|c|c|c|c|}
\hline Acceptors & $\theta_{1}$ & $\theta_{2}$ & Acceptors & $\theta_{1}$ & $\theta_{2}$ & Acceptors & $\theta_{1}$ & $\theta_{2}$ \\
\hline $1 \mathrm{a} \alpha$ & $0.01^{\circ}$ & $-0.02^{\circ}$ & $3 \mathrm{~d} \alpha$ & $0.00^{\circ}$ & $0.01^{\circ}$ & $2 \mathrm{~d} \gamma$ & $-0.51^{\circ}$ & $-18.74^{\circ}$ \\
\hline $1 b \alpha$ & $10.31^{\circ}$ & $-25.89^{\circ}$ & $3 c \alpha$ & $-9.93^{\circ}$ & $-9.52^{\circ}$ & $1 \mathrm{a} \delta$ & $0.02^{\circ}$ & $-0.03^{\circ}$ \\
\hline $1 \mathrm{co}$ & $6.72^{\circ}$ & $23.14^{\circ}$ & $1 \mathrm{a} \gamma$ & $0.02^{\circ}$ & $-0.02^{\circ}$ & $1 \mathrm{~d} \delta$ & $-0.43^{\circ}$ & $-23.72^{\circ}$ \\
\hline $1 d \alpha$ & $-1.51^{\circ}$ & $23.79^{\circ}$ & $1 d \gamma$ & $-1.80^{\circ}$ & $25.03^{\circ}$ & $2 a \delta$ & $0.01^{\circ}$ & $-0.01^{\circ}$ \\
\hline $2 \mathrm{~d} \alpha$ & $2.29^{\circ}$ & $-18.72^{\circ}$ & $2 a \gamma$ & $0.00^{\circ}$ & $0.00^{\circ}$ & $2 \mathrm{~d} \delta$ & $0.81^{\circ}$ & $-18.72^{\circ}$ \\
\hline
\end{tabular}

表 2 受体分子最大吸收波长 $\lambda_{\max }$ 、振子强度 $f$ 、激发能 $E$ 及电子重组能 $\lambda_{\mathrm{e}}$

Table 2 The maximum absorption wavelength $\lambda_{\max }$, oscillator strength $f$, excitation energy $E$ and electronic reorganization energy $\lambda_{\mathrm{e}}$ of acceptors

\begin{tabular}{|c|c|c|c|c|c|c|c|c|c|}
\hline Acceptors & $\lambda_{\max } / \mathrm{nm}$ & $f$ & $E / \mathrm{eV}$ & $\lambda_{\mathrm{e}} / \mathrm{eV}$ & Acceptors & $\lambda_{\max } / \mathrm{nm}$ & $f$ & $E / \mathrm{eV}$ & $\lambda_{\mathrm{e}} / \mathrm{eV}$ \\
\hline $1 \mathbf{a} \alpha$ & $520(448)^{a}, 448^{b}$ & $1.27(1.41)$ & $2.38(2.77)$ & 0.271 & $1 d \alpha$ & $605(502)$ & $1.77(1.98)$ & $2.05(2.47)$ & 0.274 \\
\hline $1 \mathrm{a} \beta$ & $502(430)$ & $1.26(1.50)$ & $2.47(2.88)$ & 0.274 & $1 d \boldsymbol{d} \beta$ & $589(487)$ & $1.78(2.07)$ & $2.11(2.55)$ & 0.271 \\
\hline $1 \mathrm{a} \gamma$ & $549(461)$ & $0.96(1.23)$ & $2.26(2.69)$ & 0.185 & $1 d \gamma$ & $652(530)$ & $1.36(1.72)$ & $1.90(2.34)$ & 0.186 \\
\hline $1 \mathrm{a} \delta$ & $721(631)$ & $0.63(0.68)$ & $1.72(2.12)$ & 0.210 & $1 \mathrm{~d} \delta$ & $847(757)$ & $0.89(0.83)$ & $1.46(1.86)$ & 0.216 \\
\hline $2 \mathbf{a \alpha}$ & $560(477)$ & $1.32(1.52)$ & $2.22(2.6)$ & 0.283 & $2 d \alpha$ & $628(516)$ & $1.939(2.15)$ & $1.98(2.40)$ & 0.252 \\
\hline $2 a \beta$ & $543(461)$ & $1.35(1.61)$ & $2.28(2.69)$ & 0.282 & $2 d \beta$ & $613(502)$ & $1.94(2.25)$ & $2.02(2.47)$ & 0.247 \\
\hline $2 \mathbf{a} \gamma$ & $598(497)$ & $1.07(1.38)$ & $2.07(2.5)$ & 0.189 & $2 \mathrm{~d} \gamma$ & $679(546)$ & $1.5(1.89)$ & $1.83(2.27)$ & 0.174 \\
\hline $2 \mathbf{a} \delta$ & $776(686)$ & $0.70(0.69)$ & $1.60(2.0)$ & 0.21 & $2 \mathrm{~d} \delta$ & $871(789)$ & $1.01(0.88)$ & $1.42(1.82)$ & 0.196 \\
\hline
\end{tabular}

${ }^{a}$ The parenthesis values obtained at the CAM-B3LYP/6-311G(d,p) level of theory; ${ }^{b}$ The experimental values from reference [15]. 
表 3 溶剂对受体分子最大吸收波长 $\lambda_{\text {max }}$ 、振子强度 $f$ 、激发能 $E$ 及电子重组能 $\lambda_{\mathrm{e}}$ 的影响

Table 3 Solvent effects on the maximum absorption wavelength $\lambda_{\max }$, oscillator strength $f$, excitation energy $E$ and electronic reorganization energy $\lambda_{\mathrm{e}}$

\begin{tabular}{|c|c|c|c|c|c|c|}
\hline Acceptors & $\theta_{1} / \theta_{2}\left(^{\circ}\right)$ & LUMO/HOMO(eV) & $\lambda_{\max } / \mathrm{nm}$ & $f$ & $E / \mathrm{eV}$ & $\lambda_{\mathrm{e}} / \mathrm{eV}$ \\
\hline \multirow{3}{*}{$1 \mathrm{a} \gamma$} & $0.0 / 0.0$ & $-3.99 /-6.50$ & $549(461)^{c}$ & $0.96(1.23)$ & $2.26(2.69)$ & 0.185 \\
\hline & $-26.5 /-7.4^{a}$ & $-3.67 /-6.36$ & $534(447)$ & $0.87(1.31)$ & $2.32(2.77)$ & 0.208 \\
\hline & $-24.5 /-6.8^{b}$ & $-3.72 /-6.38$ & $544(454)$ & $0.84(1.33)$ & $2.28(2.70)$ & 0.206 \\
\hline \multirow{3}{*}{$2 a \gamma$} & $0.0 / 0.0$ & $-4.08 /-6.36$ & $598(497)$ & $1.07(1.38)$ & $2.07(2.50)$ & 0.189 \\
\hline & $0.0 / 0.0^{a}$ & $-3.76 /-6.10$ & $594(505)$ & $1.12(1.59)$ & $2.09(2.45)$ & 0.152 \\
\hline & $0.0 / 0.0^{b}$ & $-3.82 /-6.14$ & $604(513)$ & $1.06(1.61)$ & $2.05(2.42)$ & 0.157 \\
\hline \multirow{3}{*}{$1 \mathrm{~d} \gamma$} & $-1.8 / 25.1$ & $-3.75 /-5.89$ & $652(530)$ & $1.36(1.72)$ & $1.90(2.34)$ & 0.186 \\
\hline & $-12.4 / 22.9^{a}$ & $-3.59 /-5.74$ & $666(543)$ & $1.33(1.89)$ & $1.86(2.28)$ & 0.145 \\
\hline & $-10.1 / 22.8^{b}$ & $-3.62 /-5.76$ & $676(551)$ & $1.28(1.91)$ & $1.83(2.25)$ & 0.147 \\
\hline \multirow{3}{*}{$2 \mathrm{~d} \gamma$} & $-0.5 /-18.7$ & $-3.85 /-5.90$ & $679(546)$ & $1.50(1.89)$ & $1.83(2.27)$ & 0.174 \\
\hline & $-8.4 /-14.8^{a}$ & $-3.63 /-5.69$ & $694(562)$ & $1.49(2.09)$ & $1.78(2.21)$ & 0.133 \\
\hline & $-5.4 /-16.2^{b}$ & $-3.67 /-5.72$ & $701(570)$ & $1.45(2.21)$ & $1.77(2.18)$ & 0.138 \\
\hline
\end{tabular}

${ }^{a}$ The second line values in acetone; ${ }^{b}$ The third line values in chlorobenzene; ${ }^{c}$ The parenthesis values obtained at CAM-B3LYP/6-311G(d,p) level of theory.

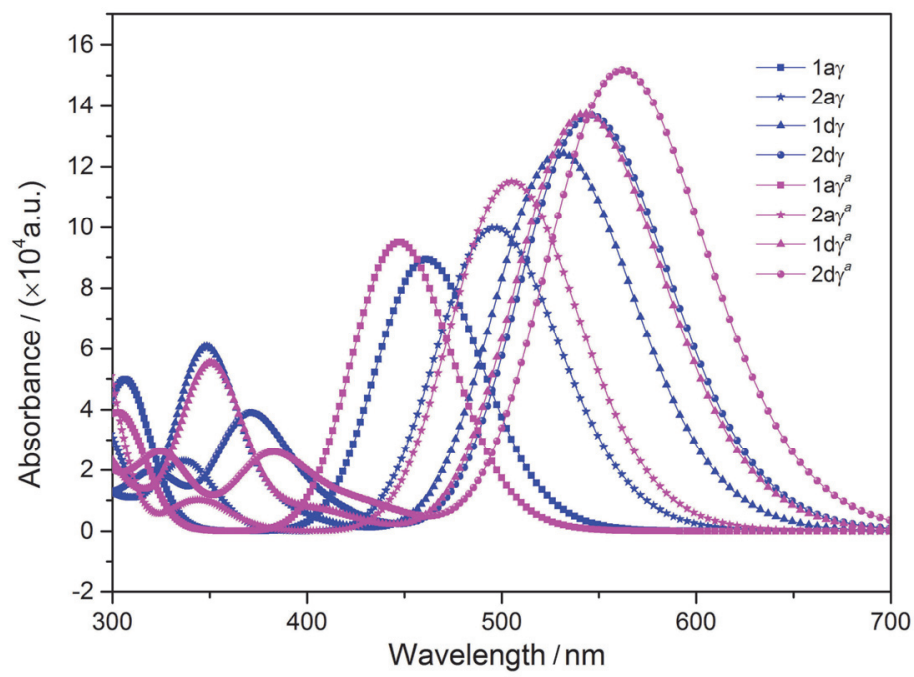

图 2 TD-CAM-B3LYP/6-311G $(\mathrm{d}, \mathrm{p})$ 水平上模拟部分苯并噻二唑衍生物在气相与丙酮溶剂中 ${ }^{a}$ 的吸收光谱

Figure 2 Simulated absorption spectra for part of benzothiadiazole derivatives at the TD-DFT CAM-B3LYP/6-311G(d,p) level $\left({ }^{a}\right.$ is in acetone)

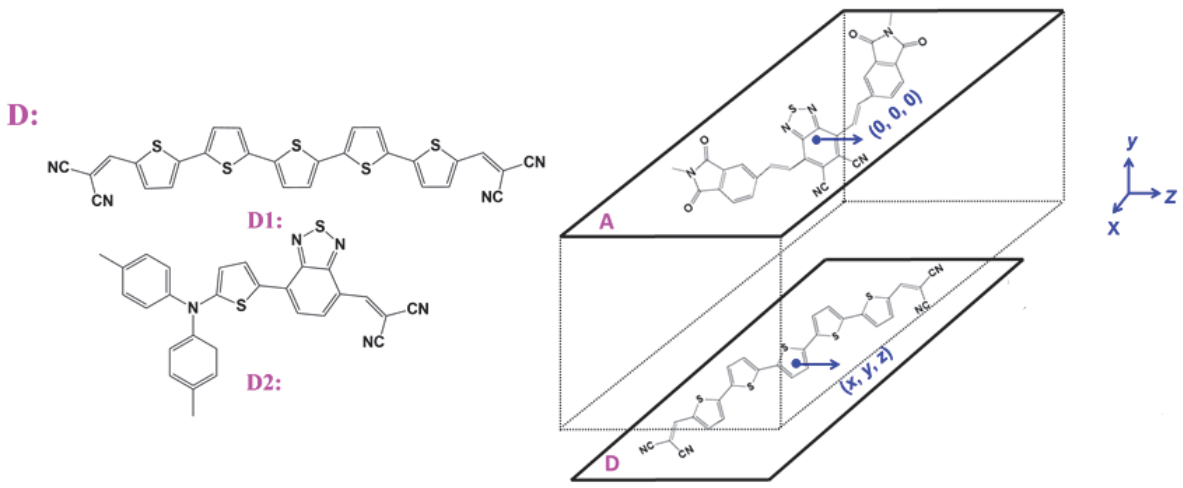

图 3 电子给体分子结构(左)及 D-A 界面构建示意图(右)

Figure 3 The molecular structure of electron donors (left) and D-A interface structure diagram (right)

有所减小.

\subsection{D-A 界面构建与载流子复合程度评估}

我们课题组先前工作预测 ${ }^{[27]}$, 将平面性好的受体 材料与非平面型给体材料搭配在一起作为有机太阳能
电池光活性层有可能获得电子迁移率高、载流子复合程 度低、开路电压 $V_{\mathrm{oc}}$ 高的 OPV 器件. 因此本文选择非平 面型电子给体分子低聚噻吩衍生物 $\mathrm{D}^{[30]}\left(E_{\mathrm{LUMO}}=\right.$ $-3.57 \mathrm{eV}, E_{\mathrm{HOMO}}=-5.90 \mathrm{eV}, \lambda_{\max }=500 \mathrm{~nm}$ )及三苯胺衍 
生物 $\mathrm{D} 2^{[31]}\left(E_{\mathrm{LUMO}}=-3.37 \mathrm{eV}, E_{\mathrm{HOMO}}=-5.46 \mathrm{eV}, \lambda_{\max }=\right.$ $638 \mathrm{~nm}$ )分别与受体分子 $\mathbf{1 a} \boldsymbol{\gamma}, \mathbf{2 a} \boldsymbol{\gamma}, \mathbf{1 d} \boldsymbol{\gamma}$ 与 $\mathbf{2 d} \boldsymbol{\gamma}$ 搭配, 构建 D-A 界面(图 3). 采用我们文献[27]所用的结合能扫描方 法确定 D-A 界面分子的相对位移, 其结果列于表 4. 其 中 D1-2a $\gamma$ 复合物结合能 $\Delta E_{\mathrm{b}}$ 随给一受体分子相对坐标的 变化示于图 4. 结合图 4 与表 4 可看出, 图 4 示出当分 子面间距 $y$ 为 $3.6 \AA$ 时, 堆积位移 $(x, z)$ 为 $(0.0,-0.8)$, 对 应结合能 $\Delta E_{\mathrm{b}}$ 较大. 当受体分子 $\mathrm{D} 1$ 与给体 $\mathbf{2 a} \boldsymbol{\gamma}$ 组合时 结合能出现多个极大点(能量极小点), 说明实际条件下 可能存在多种不同位移的堆积形式.

激子的有效分离是形成载流子的先决条件, D-A 分 子的 LUMO 能级差 $\Delta E_{\mathrm{L}}$ 是衡量激子分离效率 $\eta_{\mathrm{CT}}$ 的主要 参数, 一般要求 $\Delta E_{\mathrm{L}}$ 大于 $0.3 \mathrm{eV}$, 以保证激子有效分 离 $^{[6]} .4$ 个受体分子与 2 个给体分子共有 8 种组合方式, 其中 D1-1d $\gamma$ 与 D1-2d $\gamma$ 这 2 种组合不满足 $\Delta E_{\mathrm{L}}$ 大于 0.3 $\mathrm{eV}$ 的要求, 不能保证激子有效分离, 因此这 2 种组合在 后续研究中将不予考虑. 表 4 列出了 6 种合理组合界面 的位移信息及采用孤立轨道法计算得到 $\mathrm{HOMO}^{\mathrm{D}}$ 与
$\mathrm{LUMO}^{\mathrm{A}}$ 之间的电子耦合 $V_{\text {if. }}$ 从表 4 数据看出, 平面性 较好的给体 $\mathrm{D} 2$ 与 4 个受体分子组合时, $V_{\text {if }}$ 值在 $30 \mathrm{meV}$ 左右. 而非平面型给体 D1 与 2 个受体分子组合 D1-1a 和 D1-2a $\gamma$ 的 $V_{\text {if }}$ 为零, 界面复合可能性极小. 从图 5 下 部示出的 D1-2a $\gamma$ 组合前线轨道轮廓看出, $\mathrm{HOMO}^{\mathrm{D}}$ 与 $\mathrm{LUMO}^{\mathrm{A}}$ 相位错位, 轨道重叠非常弱, 电子耦合 $V_{\text {if }}$ 为零. 位移数据显示, D1 与 $\mathbf{1 a} \boldsymbol{\gamma}$ 及 $\mathbf{2} \mathbf{a} \boldsymbol{\gamma}$ 组合时, 稳定构型所处 的堆积位移 $(x, z)$ 均为 $(0.0,-0.8)$, 几乎保持在几何中心 位置. 从图 5 示出的 D1-2a 界面分子相对位置可推知, 这种组合位移有利于形成较强的 $\mathrm{C}-\mathrm{H} \cdots \mathrm{N}, \mathrm{C}-\mathrm{H} \cdots \mathrm{O}$ 及 $\mathrm{C}-\mathrm{H} \cdots \mathrm{S}$ 分子间氢键. 这一结果提示我们在设计分 子时可以适当引入 $\mathrm{N}, \mathrm{O}, \mathrm{S}$ 等杂原子通过分子间氢键来 调节分子的堆积方式, 尽可能降低 $\mathrm{HOMO}^{\mathrm{D}}$ 与 $\mathrm{LUMO}^{\mathrm{A}}$ 轨道重叠, 达到减小界面复合的目的. 这一结果再次证 实我们前文 ${ }^{[27]}$ 提出的观点是合理的, 即将平面性好的 受体材料与非平面型给体材料搭配作为有机太阳能电 池光活性层材料, 有可能达到降低界面复合、减小光电 压损失和提高开路电压的目的.

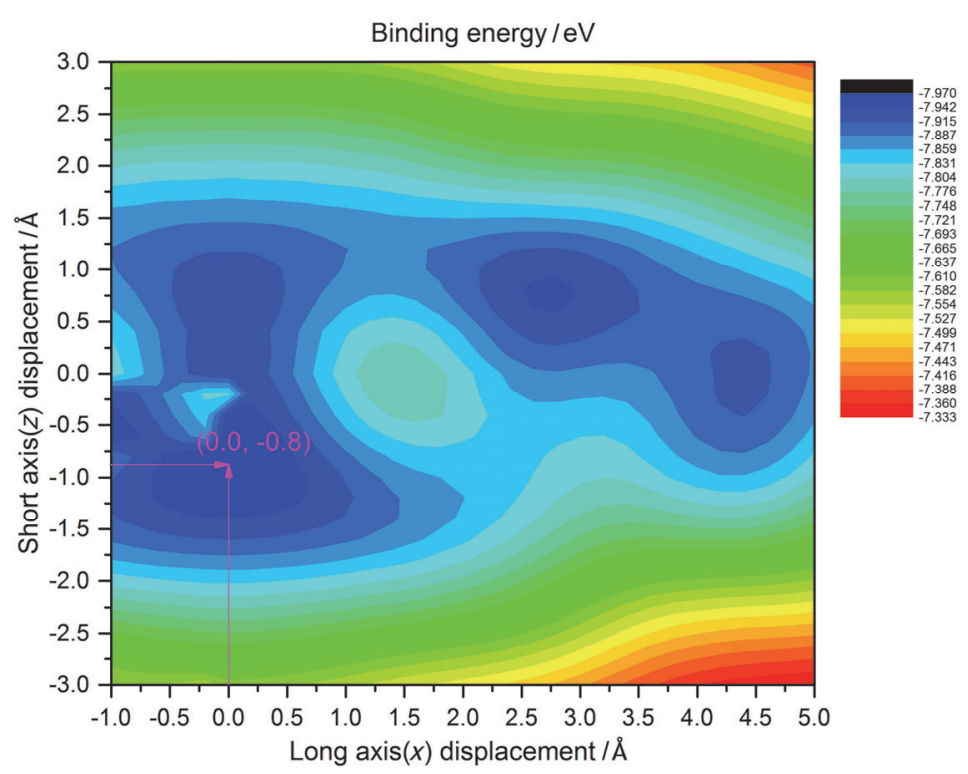

图 4 B3LYP-D3/6-311G(d,p)水平上获得 D1-2a $\gamma$ 组合结合能随界面堆积位移 $(x, z)$ 变化趋势

Figure 4 Landscapes of binding energies (B3LYP-D3/6-311G(d,p)) for D1-2a $\gamma$ combination along $x$ and $z$ axes

表 4 D-A 界面位移 ${ }^{a}$ 、电子耦合 $V_{\mathrm{if}}{ }^{b}$ 、给-受体分子 LUMO 能级差 $\Delta E_{\mathrm{L}}$ 及理论预测开路电压 $V_{\mathrm{oc}}$

Table 4 D-A interface scanning result ${ }^{a}$, the electronic coupling $V_{\text {if }}^{b}$, the difference of LUMO energy level $\Delta E_{\mathrm{L}}$ and the prediction of open circuit voltage $V_{\mathrm{oc}}$

\begin{tabular}{|c|c|c|c|c|c|c|c|}
\hline \multirow{2}{*}{ No. } & \multirow{2}{*}{ D-A } & \multicolumn{3}{|c|}{ Coordinates $(\AA)$} & \multirow{2}{*}{$V_{\mathrm{if}} / \mathrm{meV}$} & \multirow{2}{*}{$\Delta E_{\mathrm{L}} / \mathrm{eV}$} & \multirow{2}{*}{$V_{\mathrm{oc}} / \mathrm{eV}$} \\
\hline & & $x$ & $y$ & $z$ & & & \\
\hline 1 & D1-1a $\gamma$ & 0.0 & 3.6 & -0.8 & 0 & 0.42 & 1.92 \\
\hline 2 & D1-2a $\gamma$ & 0.0 & 3.6 & -0.8 & 0 & 0.51 & 1.83 \\
\hline 3 & D2-1a $\gamma$ & -0.8 & 3.8 & 0.4 & 30 & 0.62 & 1.47 \\
\hline 4 & D2-2a $\gamma$ & -0.8 & 3.7 & 0.2 & 38 & 0.71 & 1.38 \\
\hline 5 & $D 2-1 d \gamma$ & -2.2 & 3.7 & -0.2 & 27 & 0.38 & 1.71 \\
\hline 6 & D2-2d $\gamma$ & -1.0 & 3.8 & 0.4 & 31 & 0.48 & 1.61 \\
\hline
\end{tabular}

${ }^{a}$ DFT-D3-B3LYP/6-311G(d,p); ${ }^{b}$ DFT-B3LYP/6-311G(d,p). 


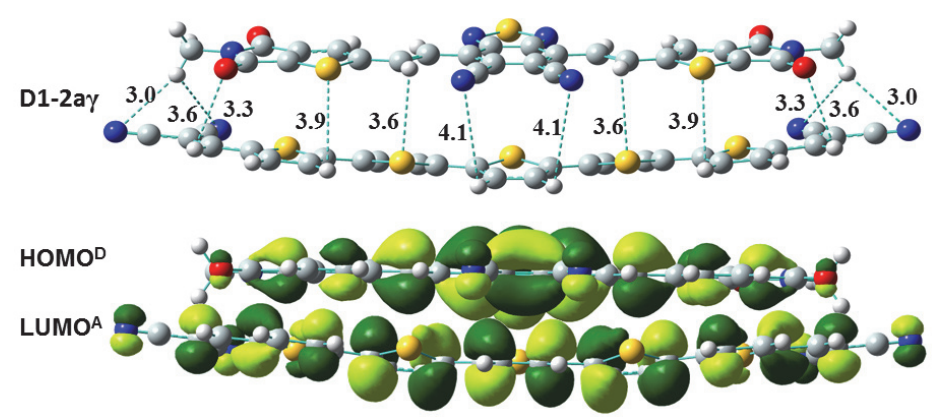

图 5 D1-2a y 界面优化构型中部分氢键示意图(单位: $\AA$, 上)及前线分子轨道轮廓图(下)

Figure 5 The diagram of hydrogen bonding in optimal configuration of D1-2a $\gamma$ interface (unit: $\AA$, top) and contour of frontier molecular orbital (below)

综合考虑 $\Delta E_{\mathrm{L}}, V_{\mathrm{if}}$, 光吸收效率及溶剂化效应等因 素, 我们认为 D1-1a $\gamma$ 及 D1-2a $\gamma$ 是可能的理想组合, 首 先受体分子的平面性好, 有利于获得良好的电子迁移 率. 其次这两种组合能有效拓宽光活性层的吸光范围, 提高光吸收效率. 同时在保证激子有效分离的基础之 上, 界面载流子复合程度小, 光电压的损失较小. 因此 D1-1a $\gamma$ 及 D1-2a $\gamma$ 组合值得实验上合成并器件化.

\section{4 结论}

以易于获得良好的电子传输性能的苯并噻二唑为 核心受体 A, 三种吸电子能力和位阻不同的共轭杂环化 合物为终端受体 $\mathrm{A}^{\prime}$, 分别以双键、噻吩、并噻吩及乙烯 噻吩为 $\pi$ 共轭桥, 设计了一系列 $\mathrm{A}^{\prime}-\pi-\mathrm{A}-\pi-\mathrm{A}^{\prime}$ 型苯并噻二 唑衍生物电子受体分子, 然后分别与低聚噻吩衍生物 D1 和三苯胺衍生物 D2 给体分子组合模拟构建有机太 阳能电池中的 D-A 界面, 通过 D-A 分子 HOMO/LUMO 能级、 $\mathrm{LUMO}$ 能级差 $\Delta E_{\mathrm{L}}$ 及 $\mathrm{HOMO}^{\mathrm{D}}$ 与 $\mathrm{LUMO}^{\mathrm{A}}$ 之间电 子耦合 $V_{\mathrm{if}}$ 等指标来评估其性能, 结果表明:

(1) 对于受体 $\mathrm{A}^{\prime}-\pi-\mathrm{A}-\pi-\mathrm{A}^{\prime}$ 型苯并噻二唑衍生物, 合 理选择取代基 $\mathrm{R}$ 对核心受体苯并噻二唑 $\mathrm{A}$ 进行修饰是 调节受体 LUMO 能级和能隙的有效方法.

(2) 将平面性好的受体材料与非平面型给体材料搭 配作为有机太阳能电池光活性层材料, 有可能达到降低 界面复合、减小光电压损失和提高开路电压的目的.

(3) D1-1a 及 D1-2a 组合在可见光和近红外区有 宽的吸收、既有较大 $\Delta E_{\mathrm{L}}$ 保证 D-A 界面激子有效分离 又有很小的 $V_{\text {if }}$ 阻止载流子复合, 是理想的给-受体分子 组合, 值得实验合成并器件化.

\section{References}

[1] Sariciftci, N. S.; Smilowitz, L.; Heeger, A. J.; Wudl, F. Science 1992, $258,1474$.

[2] Anthony, J. E.; Facchetti, A.; Heeney, M.; Marder, S. R.; Zhan, X. Adv. Mater. 2010, 22, 3876.

[3] Meng, Q. B. Acta Chim. Sinica 2015, 73, 161. (孟庆波, 化学学报, 2015, 73, 161.)

[4] Carlotto, S. J. Phys. Chem. A 2014, 118, 4808.

[5] Armin, A.; Kassal, I.; Shaw, P. E.; Hambsch, M.; Stolterfoht, M.;
Lyons, D. M.; Li, J.; Shi, Z.; Burn, P. L.; Meredith, P. J. Am. Chem. Soc. 2014, 136, 11465.

[6] Mishra, A.; Bäuerle, P. Angew. Chem. Int. Ed. 2012, 51, 2020.

[7] Walker, B.; Liu, J.; Kim, C.; Welch, G. C.; Park, J. K.; Lin, J.; Zalar, P.; Proctor, C. M.; Seo, J. H.; Bazan, G. C.; Nguyen, T.-Q. Energy Environ. Sci. 2013, 6, 952.

[8] Wang, Z.; Uemura, Y.; Zhou, Y.; Miyadera, T.; Azumi, R.; Yoshida, Y.; Chikamatsu, M. ACS Appl. Mater. Interfaces 2015, 7, 10814.

[9] Heremans, P.; Cheyns, D.; Rand, B. P. Acc. Chem. Res. 2009, 42, 1740.

[10] Reese, M. O.; Nardes, A. M.; Rupert, B. L.; Larsen, R. E.; Olson, D. C.; Lloyd, M. T.; Shaheen, S. E.; Ginley, D. S.; Rumbles, G.; Kopidakis, N. Adv. Funct. Mater. 2010, 20, 3476.

[11] Fu, Y.; Wang, F.; Zhang, Y.; Fang, X.; Lai, W.; Huang, W. Acta Chim. Sinica 2014, 72, 158. (付钰，王芳，张燕，方旭，赖文勇，黄 维, 化学学报, 2014, 72, 158.)

[12] Dou, C.; Chen, D.; Iqbal, J.; Yuan, Y.; Zhang, H.; Wang, Y. Langmuir 2011, 27, 6323.

[13] Woo, C. H.; Holcombe, T. W.; Unruh, D. A.; Sellinger, A. Chem. Mater. 2010, 22, 1673.

[14] Wolfer, P.; Schwenn, P. E.; Pandey, A. K.; Fang, Y.; Stingelin, N.; Burn, P. L.; Meredith, P. J. Mater. Chem. A. 2013, 1, 5989.

[15] Bloking, J. T.; Han, X.; Higgs, A. T.; Kastrop, J. P.; Pandey, L.; Norton, J. E.; Risko, C.; Chen, C. E.; Bredas, J.-E.; McGehee, M. D.; Sellinger, A. Chem. Mater. 2011, 23, 5484.

[16] Barone, V.; Cossi, M. J. Phys. Chem. A 1998, 102, 1995.

[17] Schlenker, C. W.; Thompson, M. E. Chem. Commun. 2011, 47, 3702.

[18] Newton, M. D.; Sutin, N. Ann. Rev. Phys. Chem. 1984, 35, 437.

[19] Barbara, P. F.; Meyer, T. J.; Ratner, M. A. J. Phys. Chem. 1996, 100, 13148.

[20] Lemaur, V.; Steel, M.; Beljonne, D.; Brédas, J.-L.; Cornil, J. J. Am. Chem. Soc. 2005, 127, 6077.

[21] Hsu, C. P. Acc. Chem. Res. 2009, 42, 509.

[22] Yang, Y. M.; Yin, S. W.; Li, L. L.; Yang, J. Y. Acta Chim. Sinica 2011，69，1991. (杨永梅，尹世伟，李兰兰，杨家瑜，化学学报， 2011, 69, 1991.)

[23] Grimme, S. WIREs Comput. Mol. Sci. 2011, 1, 211.

[24] Liu, H.; Brémond, É.; Prlj, A.; Gonthier, J. F.; Corminboeuf, C. J. Phys. Chem. Lett. 2014, 5, 2320.

[25] Guan, L.; Wang, W. L.; Shao, R.; Liu, F. Y.; Yin, S. W. J. Mol. Model. 2015, 21, 126-1.

[26] Zhan, X.; Facchetti, A.; Barlow, S.; Marks, T. J.; Ratner, M. A.; Wasielewski, M. R.; Marder, S. R. Adv. Mater. 2011, 23, 268.

[27] Shao, R.; Wang, W. L.; Yang, X. B.; Yin, X. W. Sci. China-Chem. 2016, 46, 699. (邵线, 王文亮, 杨金博, 尹世伟，中国科学：化学, 2016, 46, 699.)

[28] Liu, X.; Su, S. J.; Cao, Y. Polymer Bulletin 2014, 12, 68. (刘欣, 苏 仕健，曹镛，高分子通报，2014，12,68.)

[29] Nalwa, H. S. Handbook of Advanced Electronic and Photonic Materials and Device, Academic San Diego, CA, 2001.

[30] Fitzner, R.; Reinold, E.; Mishra, A. Adv. Funct. Mater. 2011, 21, 897.

[31] Lin, L. Y.; Chen, Y. H.; Huang, Z. Y.; Lin, H. W.; Chou, S. H.; Lin, F.; Chen, C. W.; Liu, Y. H.; Wong, K. T. J. Am. Chem. Soc. 2011, 133,15822 . 